\title{
Assessment of nutritional status in laparoscopic gastrectomy for gastric cancer
}

\author{
Young-Gil Son, In Gyu Kwon, Seung Wan Ryu \\ Department of Surgery, Keimyung University School of Medicine, Daegu, Korea \\ Contributions: (I) Conception and design: All authors; (II) Administrative support: All authors; (III) Provision of study material or patients: All authors; \\ (IV) Collection and assembly of data: All authors; (V) Data analysis and interpretation: All authors; (VI) Manuscript writing: All authors; (VII) Final \\ approval of manuscript: All authors. \\ Correspondence to: Seung Wan Ryu. Department of Surgery, Keimyung University School of Medicine, Daegu, Korea. Email: gsman@dsmc.or.kr.
}

\begin{abstract}
Malnutrition is very common in gastric cancer patients and can be detected in up to $85 \%$ of patients with gastric cancer. Malnutrition is associated with increased morbidity and mortality, prolonged hospital stay, poor treatment tolerance, and lower survival rate. Malnutrition also has an impact on quality of life. The early detection of nutritional risk with appropriate nutritional care can significantly reduce patient's postoperative morbidity and mortality. Because there is no gold standard tool, appropriate tools should be selected and applied depending on one's institutional conditions. And, it is recommended that nutritional assessment should be achieved for every patient at pre/post-operative period.
\end{abstract}

Keywords: Nutritional assessment; malnutrition; gastric cancer

Received: 10 February 2017; Accepted: 13 September 2017; Published: 28 October 2017.

doi: $10.21037 / \operatorname{tgh} .2017 .09 .08$

View this article at: http://dx.doi.org/10.21037/tgh.2017.09.08

\section{Introduction}

Gastric cancer is the fifth most common cancer and the third leading cause of cancer deaths worldwide (1). The incidence of malnutrition in gastric cancer patient is reported from $65 \%$ to $85 \%$ depending on the methods used to identify malnutrition $(1,2)$. Malnutrition tends to deteriorate during the hospitalization and may be exacerbated by treatment and disease itself $(3,4)$.

Since gastric cancer surgery is one of the high-risk major procedures for nutritional derangement, the benefit from nutrition therapy has been documented in the field of gastric cancer surgery (5). Malnutrition is associated with increased morbidity and mortality, prolonged hospital stay, poor treatment tolerance, and lower survival rate. Malnutrition also affect adversely on quality of life $(4,6,7)$. Therefore, nutritional support is necessary in malnourished patients. Moreover, there has been stiff increase of concern about adverse effects of obesity, because over nutrition also is a category of malnutrition.

Nutritional screening and assessment are important to identify patients who are malnourished or at significant risk of malnourishment and to reduce postoperative complications via appropriate nutritional intervention. There is no single gold-standard clinical or laboratory parameter for assessment of nutritional status, so many tools have been proposed to evaluate the nutritional status (8).

The aim of this article is to introduce nutritional assessment tools and to have a perception of the importance of nutrition in patients with gastric cancer. The proper nutritional interventions could have a positive effect on patients' recovery and survival.

\section{Nutritional assessment}

Malnutrition is defined as "a state resulting from lack of intake or uptake of nutrition that leads to altered body composition and body cell mass leading to diminished physical and mental function and impaired clinical outcome from disease" (9). Malnutrition increases morbidity, mortality, length of hospital stay, and medical cost. To detect preoperative malnutrition, to provide 
appropriate nutritional support, and to reduce postoperative complications, malnutrition or a risk of malnutrition should be diagnosed. Because there is no consensus among physicians about using any single nutritional assessment tool, various tools have been proposed to assess nutritional status (10). And, a systematic approach to nutritional assessment is needed to diagnose a malnutrition using various parameters that include anthropometric data, dietary assessment, laboratory parameters, and nutritional scoring systems. Nutritional assessment tools must be simple, costeffective, reproducible in different clinical setting, able to predict those patients who need nutritional intervention, and have little inter-observer variability (11).

\section{Anthropometric measurements}

Anthropometric approaches are relatively non-invasive methods and scientific measurements of patient's body size and form. Anthropometric assessment includes measurement of weight, height, and body composition (muscle mass and fat mass).

Body weight is the simplest and the most frequently used parameter in clinical setting. Unintended weight loss is a very sensitive indicator of malnutrition (12). Weight loss of more than $5 \%$ in 1 month or $10 \%$ in 6 months before hospitalization has been shown to be clinically significant (13).

Body mass index is used in nutritional assessment of patient and can be compared with standard cut-off points. BMI is calculated using the following formula: weight $(\mathrm{kg}) /$ height $^{2}\left(\mathrm{~m}^{2}\right)$. In recent retrospective study, underweight patients with BMI $<18.5$ and low preoperative albumin levels had a significantly decreased overall survival after gastrectomy for cancer (14).

Direct measurement of body fat or protein stores can be performed by measuring of triceps skin fold thickness (TSF) or mid-arm circumference (MAC). TSF can be measured at the midpoint between the acromion and olecranon process on the non-dominant side with a caliper. MAC can be measured at the same point as the TSF with a tape. A decline in subcutaneous fat and overall body muscle mass is a significant indicator of malnutrition and low levels of them correlate well with morbidity and mortality (15).

\section{Dietary intake assessment}

A thorough dietary history including current intake, previous intake, and any recent changes is necessary (16).
Dietary intake assessment using either 3-7-day food diaries or 24-hour dietary recall enables to calculate the patient's energy and protein intake. Various factors such as gastrointestinal tract obstruction, anorexia, oral ulcer and chemotherapeutic agents can decrease dietary intake in patients with gastric cancer.

\section{Laboratory parameters}

Serum hepatic protein (albumin, prealbumin, and transferrin) levels have historically been used to determine the nutritional status. However, recent studies showed that these proteins are not indicators of nutritional status, because these proteins decrease in response to infection, injury, or inflammation $(15,17)$.

Albumin is an excellent prognostic indicator, with values of less than $3.0 \mathrm{~g} / \mathrm{dL}$ associated with poor surgical outcomes. However, albumin has been considered as an unreliable nutritional indicator because its half-life is about 20 days and it is a negative acute phase protein, decreasing in response to inflammation.

Prealbumin appears to be a more reliable marker of nutritional status because of shorter half-life (2 days) than albumin. But, prealbumin is also a negative acute phase protein, therefore considered as an unreliable indicator.

Total lymphocyte count is another serum marker for determining nutritional status. Levels $<1,500 / \mathrm{mm}^{3}$ correlate with malnutrition, and those $<900 / \mathrm{mm}^{3}$ reflects severe malnutrition (15).

\section{Nutritional scores}

Overall nutritional status can be assessed through a combination of variables including historical, physical, and laboratory data. Various scoring systems have been developed and validated in different clinical setting. Validated nutrition scoring systems include the malnutrition universal screening tool, nutritional risk screening, nutritional risk index, subjective global assessment (SGA), and mini nutritional assessment (MNA) (18) (Table 1).

Malnutrition universal screening tool (MUST), which is recommended by ESPEN as the preferred screening tool for patients in the community, has been developed to detect protein-energy malnutrition and the risk of developing malnutrition by using three independent criteria: (I) current weight status using BMI; (II) unintentional weight loss; and (III) acute disease effect on nutritional intake (19). It is categorized as low risk (score $=0$ ), medium risk (score 
Table 1 Instruments for nutritional screening and assessment Nutritional screening tools

Malnutrition universal screening tool (MUST)

Nutrition risk screening 2002 (NRS 2002)

Nutritional risk index (NRI)

Prognostic nutritional index (PNI)

Malnutrition screening tool (MST)

Nutritional assessment tools

Subjective global assessment (SGA)

Patient-generated subjective global assessment (PG-SGA)

Mini nutritional assessment (MNA)

$=1$ ), and high risk (score $\geq 2$ ). And, this method provides a management guideline according to the risk of malnutrition.

Nutrition risk screening 2002 (NRS 2002) is recommended by ESPEN as the preferred screening tool for hospitalized patients (mixed population). Of all the screening tools, the NRS-2002 is the best predictor of postsurgical complications (20). NRS-2002 classifies the patient's nutritional status based on BMI, recent weight loss, recent change in dietary intake, and disease severity. And, patients are scored in each component according to whether they are absent, mild, moderate, or severe. Patients with a total score of $\geq 3$ are classified as nutritionally at risk (21).

Nutritional risk index (NRI) was developed in 1991 for assessment of the efficacy of perioperative total parenteral nutrition in malnourished patients undergoing major thoracic or abdominal surgery (22). NRI is calculated according to the following formula: $1.159 \times$ serum albumin level $(g / L)+0.417 \times($ current weight/usual weight $) \times 100$. It is categorized as no nutrition risk (score $>100$ ), borderline malnourished ( $>97.5$ ), mildly malnourished (83.5 to 97.5 ), or severely malnourished $(<83.5)$.

SGA, which is recommended by the ASPEN, is a nutritional assessment tools based on features of the history (weight change, dietary intake change, gastrointestinal symptoms, functional capacity, and disease severity) and physical examination (23). It is widely employed, is easy to implement, and can predict patients at risk for malnutrition (20). SGA ratings are: (A) well nourished; (B) moderate or suspected malnutrition; and (C) severe malnutrition.

Patient-generated SGA (PG-SGA) was adapted from SGA and developed specifically to assess malnutrition for cancer patients $(11,24)$. This self-administered SGA consists of weight, food intake, symptoms, activities and function, disease and its relation to nutritional requirements, and physical examination. It was designed that the first four sections can be completed by the patient, and remaining portions is performed by the clinician.

MNA is a rapid and efficient assessment tool for evaluating the nutritional status of the elderly. It is composed of 18 items, which include anthropometric assessment, general assessment, dietary assessment, and subjective assessment (25). The scoring for each part categorizes: (I) well-nourished; (II) at risk for malnutrition; and (III) malnourished.

\section{Clinical/practical guidelines}

The ESPEN guidelines suggest a guidance to define severe malnutrition (26). Preoperative nutritional support is recommended for patients at severe nutritional risk, defined by the presence of at least one of the following criteria: (I) weight loss $>10-15 \%$ within 6 months; (II) $\mathrm{BMI}<18.5$; (III) SGA grade C; and (IV) serum albumin $<30 \mathrm{~g} / \mathrm{L}$ (with no evidence of hepatic or renal dysfunction).

The ASPEN guidelines for diagnosing malnutrition were proposed in 2009 (12). The identification of two or more of the following six characteristics is recommended for diagnosis of malnutrition: (I) insufficient energy intake; (II) weight loss; (III) loss of muscle mass; (IV) loss of subcutaneous fat; (V) localized or generalized fluid accumulation that may sometimes mask weight loss; and (VI) diminished functional status as measured by hand-grip strength.

In the French clinical guidelines (27), the presence of even one of following three criteria is proposed to define malnutrition: (I) a $\mathrm{BMI} \leq 18.5$ or $\mathrm{BMI}<21$ in a patient older than 70; (II) recent weight loss of more than $10 \%$; and (III) a serum albumin level $<3.0 \mathrm{mg} / \mathrm{dL}$ independent of C-reactive protein.

\section{Perioperative nutritional support}

Nutritional care plan should be made for patients who are malnourished or at significant risk of malnourishment. Nutrition or nutrients can be provided by orally (regular diet, therapeutic diet, or oral nutritional supplements), by enteral feeding, or by parenteral nutrition to treat malnutrition (9). These strategies should be individualized for each patient. It was proposed that patients consuming at least $75 \%$ of nutritional requirements should receive counseling or dietetic advice, $50-75 \%$ should receive oral supplementation, and $<50 \%$ should be given enteral 
nutrition via tube feeding (3). Parenteral nutrition should be considered when energy intake cannot be met to caloric requirement via the enteral route.

For severely malnourished patients, the surgery should be delayed and nutritional intervention must be started immediately and continued preoperatively for at least 7 to 10 days. And, immune modulating substrates (arginine, glutamine, $\omega-3$ fatty acid, nucleotides, and antioxidant micronutrients) are recommended for 5-7 days in cancer patients undergoing upper major abdominal surgery (26). Preoperative intake of these immunonutrients reduces postoperative infectious complications and length of hospital stay after surgery, particularly in undernourished patients.

Recently, the enhanced recovery after surgery (ERAS) protocols have been implemented at most surgical fields. Of the ERAS protocols, there are several items regarding perioperative nutrition, such as preoperative fasting, preoperative carbohydrate loading, and early initiation of oral nutrition postoperatively (28). The American Society of Anesthesiologists guidelines recommend intake of clear fluids up to 2 hours before induction of anesthesia and solids up to 6 hours (29). Preoperative carbohydrate-rich drink before surgery has been determined to be safe and reduced hunger, thirst, anxiety, length of stay, and postoperative insulin resistance (18). Early postoperative oral feeding is feasible and safe, as well as associated with shorter hospital length of stay without increase of postoperative complications $(30,31)$.

\section{Conclusions}

Nutritional screening and assessment for malnutrition must be performed systemically and must be based on a combination of various parameters not a single parameter. Identifying the malnourished or the nutritionally at risk patients and nutritional supplementation to these patients is critical for patient's management. The early detection of nutritional risk with appropriate nutritional care can significantly reduce patient's postoperative morbidity and mortality. Because there is no gold standard tool, it is recommended that the most appropriate tool depending on one's institute is applied to every patient preoperatively for nutritional assessment.

\section{Acknowledgements}

Funding: This work was supported by the Keimyung University Research Grant of 2015 and 2016.

\section{Footnote}

Conflicts of Interest: The authors have no conflicts of interest to declare.

\section{References}

1. Rosania R, Chiapponi C, Malfertheiner P, et al. Nutrition in Patients with Gastric Cancer: An Update. Gastrointest Tumors 2016;2:178-87.

2. Gavazzi C, Colatruglio S, Sironi A, et al. Importance of early nutritional screening in patients with gastric cancer. Br J Nutr 2011;106:1773-8.

3. Mariette C, De Botton ML, Piessen G. Surgery in esophageal and gastric cancer patients: what is the role for nutrition support in your daily practice? Ann Surg Oncol 2012;19:2128-34.

4. Leuenberger M, Kurmann S, Stanga Z. Nutritional screening tools in daily clinical practice: the focus on cancer. Support Care Cancer 2010;18 Suppl 2:S17-27.

5. McClave SA, Kozar R, Martindale RG, et al. Summary points and consensus recommendations from the North American Surgical Nutrition Summit. JPEN J Parenter Enteral Nutr 2013;37:99S-105S.

6. Fukuda Y, Yamamoto K, Hirao M, et al. Prevalence of Malnutrition Among Gastric Cancer Patients Undergoing Gastrectomy and Optimal Preoperative Nutritional Support for Preventing Surgical Site Infections. Annals Of Surgical Oncology 2015;22 Suppl 3:S778-S85.

7. Van Cutsem E, Arends J. The causes and consequences of cancer-associated malnutrition. Eur J Oncol Nurs 2005;9 Suppl 2:S51-63.

8. Jones JM. The methodology of nutritional screening and assessment tools. J Hum Nutr Diet 2002;15:59-71; quiz 73-5.

9. Cederholm T, Barazzoni R, Austin P, et al. ESPEN guidelines on definitions and terminology of clinical nutrition. Clin Nutr 2017;36:49-64.

10. Prevost V, Joubert C, Heutte N, et al. Assessment of nutritional status and quality of life in patients treated for head and neck cancer. Eur Ann Otorhinolaryngol Head Neck Dis 2014;131:113-20.

11. Ottery FD. Definition of standardized nutritional assessment and interventional pathways in oncology. Nutrition 1996;12:S15-9.

12. White JV, Guenter P, Jensen G, et al. Consensus statement: Academy of Nutrition and Dietetics and American Society for Parenteral and Enteral Nutrition: characteristics recommended for the identification and 
documentation of adult malnutrition (undernutrition). JPEN J Parenter Enteral Nutr 2012;36:275-83.

13. DeLegge MH, Drake LM. Nutritional assessment. Gastroenterol Clin North Am 2007;36:1-22, v.

14. Ejaz A, Spolverato G, Kim Y, et al. Impact of body mass index on perioperative outcomes and survival after resection for gastric cancer. J Surg Res 2015;195:74-82.

15. Bharadwaj S, Ginoya S, Tandon P, et al. Malnutrition: laboratory markers vs nutritional assessment. Gastroenterol Rep (Oxf) 2016;4:272-80.

16. Davies $M$. Nutritional screening and assessment in cancer-associated malnutrition. Eur J Oncol Nurs 2005;9 Suppl 2:S64-73.

17. Fuhrman MP, Charney P, Mueller CM. Hepatic proteins and nutrition assessment. J Am Diet Assoc 2004;104:1258-64.

18. Miller KR, Wischmeyer PE, Taylor B, et al. An evidencebased approach to perioperative nutrition support in the elective surgery patient. JPEN J Parenter Enteral Nutr 2013;37:39S-50S.

19. Stratton RJ, Hackston A, Longmore D, et al. Malnutrition in hospital outpatients and inpatients: prevalence, concurrent validity and ease of use of the 'malnutrition universal screening tool' ('MUST') for adults. British Journal of Nutrition 2004;92:799.

20. Lawson CM, Daley BJ, Sams VG, et al. Factors that impact patient outcome: nutrition assessment. JPEN J Parenter Enteral Nutr 2013;37:30S-8S.

21. Kondrup J, Rasmussen HH, Hamberg O, et al. Nutritional risk screening (NRS 2002): a new method based on an analysis of controlled clinical trials. Clin Nutr 2003;22:321-36.

22. Veterans Affairs Total Parenteral Nutrition Cooperative Study Group. Perioperative total parenteral nutrition in surgical patients. N Engl J Med 1991;325:525-32.

23. Detsky AS, McLaughlin JR, Baker JP, et al. What is subjective global assessment of nutritional status? JPEN J

doi: 10.21037/tgh.2017.09.08

Cite this article as: Son YG, Kwon IG, Ryu SW. Assessment of nutritional status in laparoscopic gastrectomy for gastric cancer. Transl Gastroenterol Hepatol 2017;2:85.
Parenter Enteral Nutr 1987;11:8-13.

24. Bauer J, Capra S, Ferguson M. Use of the scored PatientGenerated Subjective Global Assessment (PG-SGA) as a nutrition assessment tool in patients with cancer. Eur J Clin Nutr 2002;56:779-85.

25. Guigoz Y, Vellas B, Garry PJ. Assessing the nutritional status of the elderly: The Mini Nutritional Assessment as part of the geriatric evaluation. Nutr Rev 1996;54:S59-65.

26. Weimann A, Braga M, Harsanyi L, et al. ESPEN Guidelines on Enteral Nutrition: Surgery including organ transplantation. Clin Nutr 2006;25:224-44.

27. Chambrier C, Sztark F, Société Francophone de nutrition clinique et métabolisme (SFNEP), et al. French clinical guidelines on perioperative nutrition. Update of the 1994 consensus conference on perioperative artificial nutrition for elective surgery in adults. J Visc Surg 2012;149:e325-36.

28. Mortensen K, Nilsson M, Slim K, et al. Consensus guidelines for enhanced recovery after gastrectomy: Enhanced Recovery After Surgery (ERAS(R)) Society recommendations. Br J Surg 2014;101:1209-29.

29. Committee ASoA. Practice guidelines for preoperative fasting and the use of pharmacologic agents to reduce the risk of pulmonary aspiration: application to healthy patients undergoing elective procedures: an updated report by the American Society of Anesthesiologists Committee on Standards and Practice Parameters. Anesthesiology 2011;114:495-511.

30. Liu X, Wang D, Zheng L, et al. Is early oral feeding after gastric cancer surgery feasible? A systematic review and meta-analysis of randomized controlled trials. PLoS One 2014;9:e112062.

31. Willcutts KF, Chung MC, Erenberg CL, et al. Early Oral Feeding as Compared With Traditional Timing of Oral Feeding After Upper Gastrointestinal Surgery: A Systematic Review and Meta-analysis. Ann Surg 2016;264:54-63. 